\title{
Narrowband perfect metasurface absorber based on impedance matching
}

\author{
Muhammad Ali Butt, ${ }^{* 1,3}$, Nikolay Lvovich Kazanskiy ${ }^{1,2}$ \\ ${ }^{1}$ Department of Technical Cybernetics, Samara National Research University, 34 Moskovkoye Shosse, Samara \\ 443086, Russia \\ ${ }^{2}$ Institute of RAS-Branch of the FSRC "Crystallography and Photonics" RAS, 151 Molodogvardeiskaya, Samara \\ 443001, Russia \\ ${ }^{3}$ Institute of Microelectronics and Optoelectronics, Warsaw University of Technology, Koszykowa 75, 00-662 \\ Warszawa, Poland
}

Received August 01, 2020; accepted September 26, 2020; published September 30, 2020

\begin{abstract}
We presented a numerical investigation of a metamaterial narrowband perfect absorber conducted via a finite element method based on commercially available COMSOL software. The periodic array of silicon meta-atoms (MAs) are placed on an $80 \mathrm{~nm}$ thick gold layer. The broadband light at normal incidence is blocked by the gold layer and silicon MAs are used to excite the surface plasmon by scattering light through it. A maximum absorption of $95.7 \%$ is obtained at a resonance wavelength of $1137.5 \mathrm{~nm}$ due to perfect impedance matching of electric and magnetic dipoles. The absorption is insensitive to a wideangle of incidence ranging from 0 to 80 degrees. We believe that the proposed metamaterial device can be utilized in solar photovoltaic and biochemical sensing applications.
\end{abstract}

For a wide range of applications such as energy harvesting, emitters, solar photovoltaic, light modulation and colour filters, it is exceedingly attractive to have perfect absorbers [1-2]. The long-established way of realizing maximum absorption is by deploying the multilayer structures of metal/dielectric films with an overall thickness much higher than that of working wavelengths [3]. However, these bulky designs breach the current trend of scaling down photonic and optical systems to better incorporate scientific developments and industrial needs. Metasurface offers a proficient solution to these problems, as it can provide miniaturized, flexible and fast optical switching [4]. Metamaterials are artificial structures with permittivity and permeability unattainable in nature, have recently received much consideration because of their extraordinary electromagnetic response. Many unusual phenomena were accomplished with suitable designs, including superlenses, invisible cloaking, smart solar power management and optical filters, among others [5-7]. The dimensions of the metasurfaces are usually well beyond the wavelength, which replaces the need for bulk optics and is also capable of manipulating light at the nanoscale. This results in a simple regulation of amplitude, polarization and phase of incident light and enables a sub-wavelength effect.

${ }^{*}$ E-mail: ali_ciit_engineer@yahoo.com
Above and beyond, it has been shown that metamaterials can be used for effective electromagnetic absorption, and a new research field about perfect absorbers has been stimulated and investigated [8-9]. Metamaterials can be characterized by complex electric permittivity and magnetic permeability $\widetilde{\epsilon}(\omega)=\epsilon_{1}+i \epsilon_{2}$ and $\widetilde{\mu}(\omega)=$ $\mu_{1}+i \mu_{2}$, respectively. However, major attention was given to the real part of permittivity $\left(\epsilon_{1}\right)$ and permeability $\left(\mu_{1}\right)$ to produce negative refractive index materials. Nonetheless, the loss components of optical constants $\left(\epsilon_{2}\right.$ and $\mu_{2}$ ) have a future in crafting several practical applications such as perfect absorbers. It is likely to absorb both incident electric and magnetic fields by independently controlling the resonances in $\varepsilon$ and $\mu$. Due to its compact size and superior functionalities, this kind of absorber exhibits fabulous qualities over its traditional counterparts. The main motivation is to diminish reflectance through impedance matching and at the same time block transmittance by maximizing metamaterial losses.

In this work, we presented a flexible design of a metamaterial narrowband perfect absorber composed of an array of silicon meta-atoms (MAs) placed on top of a gold layer to eliminate transmission. The device design is highly attractive as the absorbance is insensitive to the incidence angle of light and the reflection almost negligible at a resonating wavelength. Silicon nanostructures are CMOS compatible, which provides low optical losses compared to plasmonic nanostructures. The device design is optimized in such a way that the reflection is diminished at the resonance wavelength following the electric and magnetic dipole impedance matching. Additionally, the scattered light from the silicon MAs excites the free electrons of the gold layer beneath and generates localized surface plasmon resonance (LSPR) [10]. The proposed metamaterial narrowband perfect absorber design is shown in Figure 1, consisting of a periodic array of silicon MAs placed on the gold layer which is deposited on a quartz substrate. 
Additional simulation volume is saved by taking into consideration a single cell to reduce the simulation time. Floquet-periodic boundary conditions are applied on four sides of the unit cell to simulate an infinite $2 \mathrm{D}$ array.

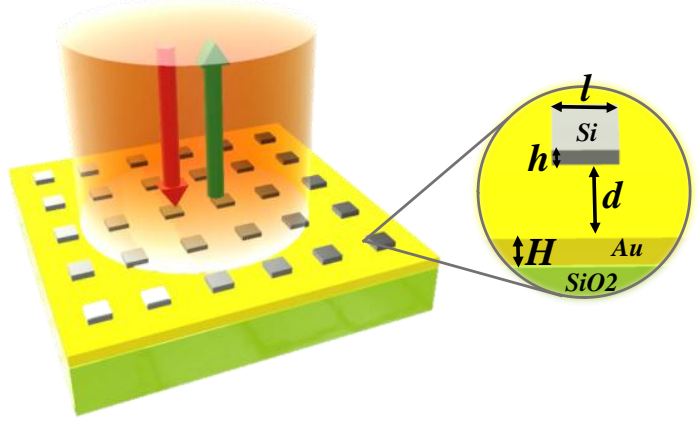

Fig. 1. 3D schematic illustration of a metamaterial narrowband perfect absorber (left). The inset (right) shows a silicon meta-atom placed on a gold layer.

As the side length and width of the silicon MAs are symmetric, we represent them as " $l$ ". The gap between the two MAs is represented as " $2 d$ ", where $d$ is the gap between MA and the boundary. As we are using a periodic boundary condition on the lateral sides of the design therefore $d$ will be automatically doubled. The height of silicon MAs and the gold layer are represented as $h$ and $H$, respectively. The gold layer $(H)$ is fixed at 80 $\mathrm{nm}$ throughout the paper, which is larger than the skin depth so it is modelled as a perfect electric conductor (PEC). The remaining domains above and below the absorber design are filled with air $(n=1.0)$. As a result, the electromagnetic (EM) wave will not be able to penetrate in the near-infrared region and the transmission will be nearly zero. The metamaterial narrowband perfect absorber is located between the source port and the output (listener) port. EM waves are emitted from the source port and measured at the listener port. Port boundary conditions are placed on the interior boundaries of the perfectly matched layers (PMLs), adjacent to the air domains which establish the transmission and reflection characteristics in terms of $S$-parameters. Where $S_{21}$ and $S_{11}$ are the transmission and reflection coefficients that can be written as [11]:

$$
S_{21}=\frac{\left(1-Z^{2}\right) \Gamma}{1-Z^{2} \Gamma^{2}}, S_{11}=\frac{\left(1-\Gamma^{2}\right) Z}{1-Z^{2} \Gamma^{2}}
$$

where $Z=\exp \left(-j k_{n} n_{e f f} L\right)=\exp \left( \pm \omega j \sqrt{\epsilon_{e f f} \mu_{e f f}} L\right)$

$L$ is the effective length, $n_{\text {eff }}$ is the effective refractive index, $\epsilon_{\text {eff }}$ and $\mu_{\text {eff }}$ are the effective permittivity and permeability. $\Gamma$ is the reflection coefficient, which can be calculated using the following expression:

$$
\Gamma=\frac{\left(Z_{o}-1\right)}{\left(Z_{o}+1\right)}
$$

where $Z_{o}$ is the relative impedance. The interior port boundaries with PMLs backing require the slit condition. PMLs are used to absorb the excited mode from the source port and any higher-order modes generated by the periodic structures. The PMLs attenuate the wave as it travels in the direction perpendicular to the PML boundary.

The resonance wavelength $\left(\lambda_{\text {res }}\right)$ and the absorbance accessible by the proposed absorber design is investigated by taking into consideration three vital parameters such as the perimeter of the silicon MAs, height of the MAs and gap between them. The maximum absorption is obtained when the electric permittivity $(\varepsilon)$ and magnetic permeability $(\mu)$ satisfy the impedance-matched condition, i.e., $\varepsilon(\omega)=\mu(\omega)$, at the operational wavelength. The absorption is calculated by using the following expression: Absorption $=1-$ Transmission - Reflection. Where transmission $\approx 0$, due to the thick gold layer deposited on the substrate. Therefore, the absorption is directly dependent on the reflection. We determined the dependence of $\lambda_{\text {res }}$ and absorption on the side length $(l)$ of the MA which is varied between $180 \mathrm{~nm}-300 \mathrm{~nm}$, while keeping $d, h$ and $H$ fixed at $350 \mathrm{~nm}, 50 \mathrm{~nm}$ and $80 \mathrm{~nm}$, respectively as shown in Fig. $2 \mathrm{a}$. It can be seen that $\lambda_{\text {res }}$ performs a redshift as $l$ increases. The maximum possible absorption of $69 \%$ is obtained at $l=260 \mathrm{~nm}$ for $\lambda_{\text {res }}=1021$ $\mathrm{nm}$. We would like to further enhance the absorbance at $l=260 \mathrm{~nm}$ by varying $h$. The remaining parameters such as $l, H$ and $d$ are fixed at $260 \mathrm{~nm}, 80 \mathrm{~nm}$ and $350 \mathrm{~nm}$, respectively. It can be seen that $\lambda_{\text {res }}$ experiences a redshift as $h$ increases. There is also a positive effect of the optimized value of $h$ on the overall absorption of the MA as shown in Fig. 2b. The optimized value of $h$ should be selected where the impedance matching condition is satisfied to obtain maximum absorption. A maximum absorption of $74.5 \%$ is obtained at $h=80 \mathrm{~nm}$ at $\lambda_{\text {res }}=1160$ $\mathrm{nm}$. In the third round of optimization, we maintained the optimized value of $l$ and $h$ to find the influence of $d$ between the MAs. The parameters such as $l, h$ and $H$ used in this simulation are set at $260 \mathrm{~nm}, 80 \mathrm{~nm}$ and $80 \mathrm{~nm}$, respectively. It can be seen from Fig. $2 \mathrm{c}$ that maximum absorption is at $95.7 \%$ when $d=300 \mathrm{~nm}$. As $d$ increases the absorption decreases whereas $\lambda_{\text {res }}$ is slightly affected and stays in the range of $1137 \div 1164 \mathrm{~nm}$ as $d$ increases from $300 \mathrm{~nm}$ to $400 \mathrm{~nm}$. The relationship of absorption, reflection and transmission spectra of the proposed metamaterial narrowband perfect absorber concerning the wavelength spectrum is shown in Fig. 2 d. The optimized geometric parameters are as follows: $l=260 \mathrm{~nm}, h=80$ $\mathrm{nm}, d=300 \mathrm{~nm}$ and $H=80 \mathrm{~nm}$. The transmittance and reflectance are negligible at $\lambda_{\text {res }}=1137.5 \mathrm{~nm}$, resulting in a maximum absorption of $95.7 \%$ as illustrated in Fig. 2 d. 

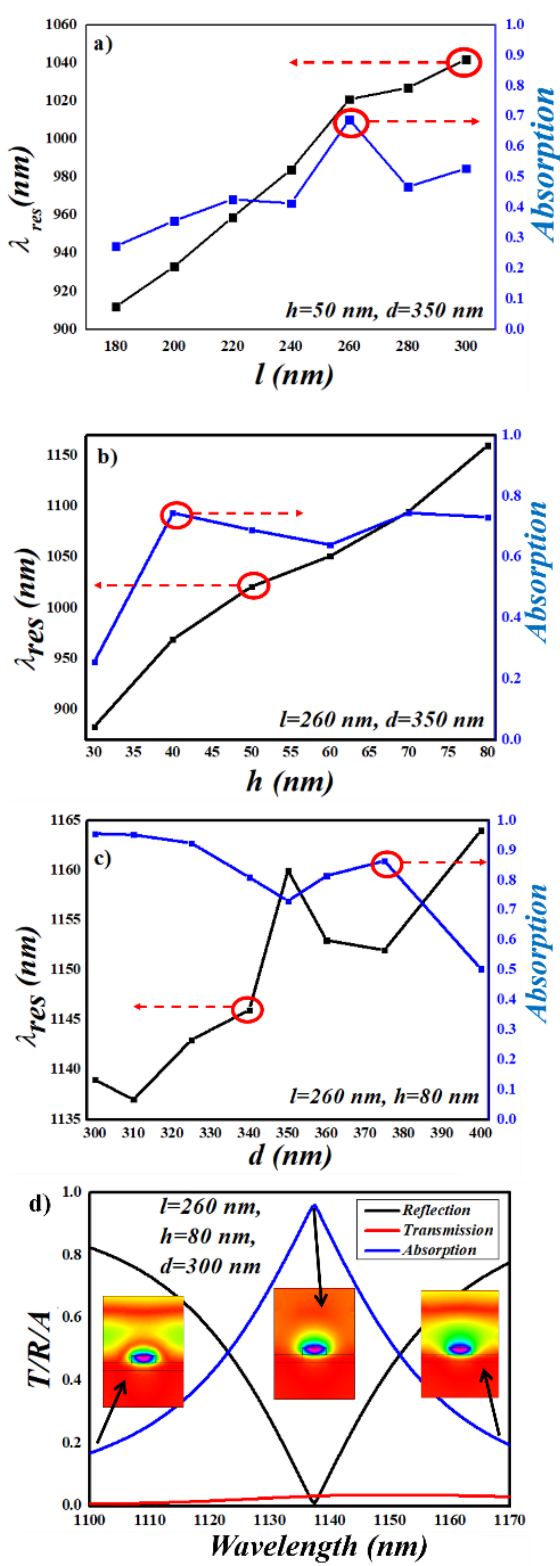

Fig. 2. Dependence of resonance wavelength and absorbance on, a) side length, b) height, c) gap of MAs. d) transmission, reflection and absorption spectrum of the proposed metamaterial narrowband perfect absorber.

The E-field distribution in a MA in the top and crosssection view at $\lambda_{\text {res }}$ is shown in Fig. 3. It is worth noting that E-field distribution is stronger on top and inside the MAs, at $\lambda_{\text {res }}=1137.5 \mathrm{~nm}$ as illustrated in Fig. 3 ( $\mathrm{a}$ and $\mathrm{b}$ ). Similarly, the H-field distribution is also plotted in top and cross-section view at $\lambda_{\text {res, }}$ as shown in Fig. 3 (c, d). Hfield is stronger between the metal and the silicon MA interface at $\lambda_{\text {res. }}$ The perfect matching of the E-field dipole and $\mathrm{H}$-field dipole results in the elimination of reflectance and provides maximum absorbance while at offresonance wavelength, the light is reflected from the surface resulting in negligible absorbance.

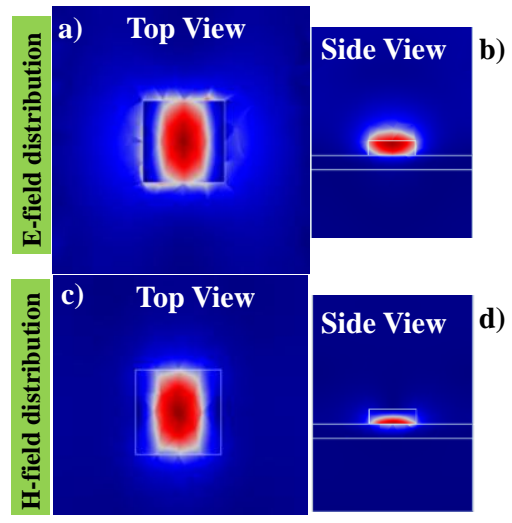

Fig. 3. E-field distribution at a) surface of the MA, b) cross-section view of the MA. H-field distribution at c) the bottom of the MA, d) crosssection view of the MA.

In conclusion, we proposed an attractive and simplified design of a perfect metamaterial absorber which consists of silicon meta-atoms periodically deposited on an $80 \mathrm{~nm}$ thick gold layer. The maximum absorption of $95.7 \%$ is obtained at a resonating wavelength of $1137.5 \mathrm{~nm}$ due to the perfect impedance matching of electric and magnetic dipoles. Another key feature of the proposed design is that the absorption is independent of the incidence angle of light and the reflection almost negligible at a resonating wavelength. The resonating wavelength of the absorber can be tuned by varying the geometric parameters of the meta-atoms. We believe that the study conducted in this work is beneficial for the development of narrowband and broadband perfect absorbers.

This work was financially supported by the Ministry of Science and Higher Education within the State assignment FSRC «Crystallography and Photonics» RAS (No. 007-GZ/Ch3363/26) for numerical calculations and Russian Science Foundation (No. 20-69-47110) for theoretical results.

\section{References}

[1] Y. Cheng, X.S. Mao, C. Wu, L. Wu, R.Z. Gong, Opt. Mat. 53, 195 (2016).

[2] S.S. Mirshafieyan, D.A. Gregory, Scien. Rep. 8, 2635 (2018)

[3] D.M. Nguyen, D. Lee, J. Rho, Scien. Rep. 7, 2611 (2017).

[4] Y. Sun, Y. Ling, T. Liu, L. Huang, AIP Advances 5, 117221 (2015).

[5] H. Chu, Q. Li, B. Liu, J. Luo, S. Sun, Z. H. Hang, L. Zhou, Y. Lai, Light: Science Appl. 7, 50 (2018).

[6] S.K. Patel, S. Charola, J. Parmar, M. Ladumor, Mat. Research Expr. 6, 086213 (2019).

[7] Q. Qian, S. Ti, C. Wang, Opt. Lett. 44, 3984 (2019).

[8] P. Yu et al., Adv. Opt. Mat. 7, 1800995 (2019).

[9] Y. J. Kim et al., Science Technol. Advan. Mater. 19, 711 (2018).

[10] N.L. Kazanskiy, S.N. Khonina, M.A. Butt, Physica E 117, 113798 (2020).

[11] H.E. Nejad, A. Mir, A. Farmani, IEEE Sensors J. 19, 4874 (2019). 